\title{
Migration of renal tumor cells depends on dephosphorylation of Shc by PTEN
}

\author{
ELKE SCHNEIDER $^{1 *}$, ROMY KEPPLER ${ }^{3 *}$, DIRK PRAWITT ${ }^{2}$, CHRISTOPH STEINWENDER $^{3}$, \\ FREDERIK C. ROOS $^{1}$, JOACHIM W. THÜROFF ${ }^{1}$, EKKEHART LAUSCH ${ }^{3}$ and WALBURGIS BRENNER ${ }^{1}$ \\ ${ }^{1}$ Department of Urology, ${ }^{2}$ Center for Pediatrics and Adolescent Medicine, University Medical Center \\ of the Johannes Gutenberg-University Mainz, Langenbeckstr. 1, D-55131 Mainz; ${ }^{3}$ Department of Pediatrics, \\ University of Freiburg, Hugstetter Strasse 49, D-79106 Freiburg, Germany
}

Received August 26, 2010; Accepted October 27, 2010

DOI: $10.3892 /$ ijo.2010.893

\begin{abstract}
The tumor suppressor PTEN is a phosphatase using FAK and Shc as direct substrates, and Akt as a key effector via PIP3. PTEN regulates cell migration and may influence metastases. We quantified PTEN in 135 clear cell renal cell carcinomas (ccRCC) by Western blot analysis and found statistically significant lower PTEN expression in patients who died, usually caused by metastases, within 5 years after surgery, compared to those surviving this time period. In athymic mice, PTEN transfected 786-O cells were injected into the tail vein and metastatic load of the lungs was quantified. We observed a strongly reduced metastatic load after PTEN transfection. For analyses of the PTEN activities, transfections with mutated PTEN genes were performed, leading to loss of lipid phosphatase activity and/or protein phosphatase activity, and of the C-terminal tail. Cell migration was analyzed in a Boyden chamber and phosphorylation of PTEN downstream targets Akt, FAK and Shc by Western blotting. 786-O cells transfected with the functional PTEN gene showed profoundly diminished migration. Transfection with a mutated PTEN isoform leading to loss of protein phosphatase activity, but not of lipid phosphatase activity, abolished this
\end{abstract}

Correspondence to: Dr Walburgis Brenner, Department of Urology, University Medical Center of the Johannes Gutenberg-University Mainz, Langenbeckstr. 1, D-55131 Mainz, Germany

E-mail: brenner@uni-mainz.de

*Contributed equally

Abbreviations: ccRCC, clear cell RCC; ECM, extracellular matrix; EGFP, enhanced green fluorescent protein; FAK, focal adhesion kinase; MAPK, mitogen activated protein kinase; PBS, phosphatebuffered saline; PDZ, PSD-95, DLG, ZO-1; PIP3, phosphatidylinositol 3,4,5-triphosphate; PTEN, phosphatase and tensin homologue deleted from chromosome 10; RCC, renal cell carcinoma; Shc, Src homology collagen; VHL, von Hippel Lindau; wtPTEN, wild-type PTEN

Key words: PTEN, migration, metastasis, renal cell carcinoma, Shc effect. Shc but not FAK seems to mediate this effect. These results show a critical role of PTEN in metastasis of RCC, depending on protein phosphatase activity via Shc. This new insight opens an alley of additional approaches complementing current cancer therapy and metastasis prediction in RCC.

\section{Introduction}

The tumor suppressor PTEN is a dual specific protein and phospholipid phosphatase ubiquitously expressed in epithelial tissue. It has been shown to affect cell survival, proliferation, apoptosis and cell motility $(1,2)$. The PTEN gene is located on chromosome 10q23.3 and includes 9 exons (3). The PTEN phosphatase comprises 403 amino acids, with an N-terminal phosphatase domain, followed by a C2 domain playing an important role in membrane localization (4). These two domains form the catalytic phospholipid and protein phosphatase unit of PTEN. The C-terminus includes a PDZ (PSD-95, DLG, ZO-1) binding domain and several phosphorylation sites (1). PTEN has been shown to bind to PDZcontaining proteins near the cell membrane, thus recruiting it into the PTEN-associated complex (5-7). Phosphorylation of PTEN in the C-terminal tail results in a conformation change, leading to a masking of the PDZ binding site (8). After dephosphorylation, PTEN is transiently activated and becomes more sensitive to protease digestion $(1,8)$.

In many advanced tumor entities, the PTEN gene is mutated or deleted $(9,10)$. Somatic point mutations are described as being associated with various tumor entities and are observed most often in endometrial carcinoma, glioblastoma and prostate carcinoma (11). Germline mutations of PTEN have been found to be distributed in all of the gene, with the exception of exon 9. Approximately one third of all mutations can be found in exon 5, which encodes for the catalytical core motif of the phosphatase domain. Some of these mutations inhibit only specific activities of the PTEN molecule, leaving other PTEN functions intact, so does the transversion c.370T $\rightarrow$ A [nomenclature of Beaudet and Tsui (12)] in exon 5, first described in endometrial carcinoma, lead to an amino acid exchange in position $124(\mathrm{C} 124 \mathrm{~S})$ that inhibits lipid and protein phosphatase activity of the enzyme (13). The transition c. $386 \mathrm{G} \rightarrow \mathrm{A}$, also located in exon 5 results in a G129E exchange 
that abrogates specifically lipid phosphatase activity of PTEN (14). Furthermore, a deletion of exon 3 markedly reduces the protein phosphatase activity and leads to an augmented localization of PTEN to the nucleus (15).

PTEN is involved in the regulation of a variety of signal transduction pathways. By dephosphorylation of PIP3 (phosphatidylinositol 3,4,5-triphosphate) in the 3-position of the inositol ring, PTEN antagonizes the PI3 kinase and inhibits the activation of Akt/PKB (16). Thereby PTEN inhibits cell proliferation and migration, and induces apoptosis $(17,18)$. By reducing the phosphorylation of Shc (Src-homology collagen), an $\mathrm{SH} 2$ binding adaptor molecule, PTEN inhibits the MAPK pathway and reduces cell proliferation. The protein phosphatase activity of PTEN is also involved in the dephosphorylation and inactivation of focal adhesion kinase (FAK). Thereby it also regulates cell proliferation and the interaction between extracellular matrix and cytoskeleton with consequences on cell motility (19). These findings suggest that PTEN dependent cell migration is regulated by the protein phosphatase and the phospholipid phosphatase activity of PTEN, although the role of PIP3-independent effects of PTEN are controversially discussed $(20,21)$. However, other mechanisms of involvement of PTEN in cell migration have also been identified. So it has been shown that over-expression of PTEN inhibited migration of outward mesodermal cells from the anterior primitive streak during chicken embryo development (18). After transfection of these cells with PTEN containing the point mutation $\mathrm{C} 124 \mathrm{~S}$, the inhibitory effect of PTEN on migration was abolished. However, the mutation G129E, that retained protein phosphatase activity, showed an inhibitory effect as effectively as wild-type PTEN (18). Microinjection of G129E mutated PTEN in PTEN null glioblastoma cells also resulted in inhibition of cell motility (22), suggesting that the protein phosphatase activity of PTEN is essential for migration of these cells.

In this report, we studied the role of PTEN in metastases of clear cell renal cell carcinoma (ccRCC). We compared the PTEN expression in ccRCC specimens with the survival rate of the patients and analyzed the metastatic activity of ccRCC cells transfected with PTEN. Our in vitro system was finally tested in a mouse model. To investigate the molecular mechanisms involved in this process in detail, we used wtPTEN and different mutated PTEN isoforms, that selectively impaired specific PTEN activities. The C124S isoform was used to generate loss of general phosphatase activity, G129E for ablation of protein phosphatase activity ( $\Delta$ exon3) for deprivation of lipid phosphatase activity (15) and the $\Delta C$ terminus for loss of PDZ binding domain and phosphorylation sites, respectively. We analyzed the effect of these isoforms on cell migration and the activity of downstream targets of PTEN.

\section{Materials and methods}

Specimens. Tissue samples were obtained under sterile conditions from 144 patients, 81 males and 53 females, median age of 65.3 years, with primary ccRCC who underwent nephrectomies at the Department of Urology of the University Medical Center of the Johannes Gutenberg University, Mainz, Germany between 1994 and 2002. Informed consent was obtained from each patient. Samples from renal cortex and tumor tissue $(\sim 5 \times 5 \times 5 \mathrm{~mm})$ were shock-frozen in liquid nitrogen and stored at $-80^{\circ} \mathrm{C}$. The diagnosis of ccRCC was based on hematoxylin and eosin sections.

In vivo analyses of metastases. The influence of PTEN in metastasis of renal cancer was investigated in vivo. The capability of kidney cancer cells to metastasize in the lung was analysed by comparing 786-O cells lacking PTEN and VHL but expressing EGFP with 786-O cells expressing wtPTEN or VHL. Real-time PCR was used for quantification of the tumor load in the lungs of athymic mice (3 animals per group) four weeks after i.v. injection of $10^{6} 786-\mathrm{O}$ cells transfected with either EGFP, wtPTEN or VHL in $200 \mu 1$ PBS. DNA was extracted using QIAamp DNA Mini Kit (Quiagen) and a 226-bp fragment of the $\alpha$-satellite region of the human chromosome 17 (sense 5'-CCT CTG ACT AAA CAG AAG CAG-3'; antisense 5'-GAG TTG AAT GCA ATC ACA G-3') was amplified from $1 \mu \mathrm{g}$ total DNA. The tumor load was quantified by referring to a standard curve generated by a serial dilution of human cells in NIH3T3 mouse fibroblasts. Animal procedures conformed to the Guide for the Care and Use of Laboratory Animals published by the National Institutes of Health. The protocol was approved by the Institutional Animal Care and Use Committee.

Cell line and cell culture. Clear cell renal carcinoma cell line 786-O, that is per se PTEN negative, was used for all experiments. These cells are characterized by a loss of the $V H L$ gene and meet all characteristics of a typical clear cell RCC (23). Cells were cultured in DMEM (Dulbecco's modified Eagle's medium, Sigma-Aldrich) with L-glutamine (Gibco), supplemented with $10 \%$ fetal calf serum (PAA Laboratories) and $0.5 \%$ penicillin/streptomycin (Pan). The cells were incubated at $37^{\circ} \mathrm{C}$ in a humidified atmosphere containing $5 \%$ $\mathrm{CO}_{2}$ in air.

Cloning of mutations and plasmid construction. In short, cDNA of wtPTEN was PCR amplified, inserted into a pcDNA3 vector and 3 ' in frame tagged with a V5 encoding domain. In addition VHL and EGFP (as an expression control) cDNAs containing the complete ORFs were each cloned directionally into a NaeI/BglII digested, alkaline phosphatase treated pCDNA3 vector (Invitrogen). Chemocompetent DH5 $\alpha$ E. coli cells were transfected with the expression plasmids including genes described and EGFP as a control. Individual clones were selected according to their resistance to ampicilline and verified by complete sequencing of the plasmid.

To produce a mutant with an inactive protein and lipid phosphatase activity, point mutation PTEN-C124S was derived from $w t P T E N, 3^{\prime}$ in frame tagged with a V5 domain, using the mutagenic oligonucleotide primer 5'-AGC AAT TCA CAG TAA AGC TGG AAA G-3' (forward) and 5'-CTT TCC AGC TTT ACT GTG ATT TGC T-3' (reverse). Point mutated PTEN-G129E was derived from wtPTEN using the mutagenic oligonucleotide primer 5'-TCA CTG TAA AGC TGG AAA GGA ACG AAC TGG TGT AAT GA-3' (forward) and 5'TCA TTA CAC CAG TTC GTT TAC AGT GA-3' (reverse). For verification of a successful point mutation, the resulting cDNAs were analysed by sequencing. 
Deletion of exon 3 ( $\Delta$ exon3) was created by modified PCR using the primer for exons 1-2 5'-TCC TTC CAT CCG CGC CGC CT-3' (forward) and 5'-TTC AAA AAG CTT ACT ACA TCA-3' (reverse). Primers for exons 4-9 were 5'-GCG AAG CTT TGC TGA AAG ACA-3' (forward) and 5'-CAC ATA GCG CCT CTG ACT GG-3'. These primers include restriction sites for the restriction endonuclease HindIII. Deletion of the $\mathrm{C}$-terminus ( $\triangle \mathrm{C}$-terminus) was performed by digestion of the 3 ' in frame V5 tagged wtPTEN-pBABEpuro with $N$ S I and BstBI and subsequent religation. This process deletes a 201-bp fragment including the PDZ binding domain and C-terminal phosphorylation sites.

All PTEN constructs thus were tagged with a V5 domain for specific detection by a V5 specific antibody (Serotec). After cutting of the pCDNA3 plasmids containing EGFP, VHL or the V5 tagged PTEN-variants with BamHI and PmeI, the purified fragments were directionally inserted into a compatibly linearized and alkaline phosphatase treated pBABE puro vector. Chemocompetent $E$. coli $\mathrm{DH} 5 \alpha$ cells were transfected with the expression plasmids including genes described and $\mathrm{DH} \alpha 5$ clones were picked for their resistance to ampicilline.

Production of recombinant retroviruses and retroviral infections. To obtain recombinant retroviruses, BOSC-23 ecotropic packaging cells (24) maintained in DMEM supplemented with $10 \%$ FCS in humidified $5 \%$ carbon dioxide atmosphere were transfected as described (24) using the pBABE retroviral vectors coding for either VHL, EGFP, the V5 tagged PTEN versions or the empty vector. In short, $5 \times 10^{6}$ BOSC- 23 cells were plated onto $10-\mathrm{cm}$ tissue culture dishes in DMEM supplemented with $10 \%$ FCS and grown for $24 \mathrm{~h}$. One $\mu \mathrm{g}$ of each expression plasmid/10-cm dish was transfected with the calcium phosphate precipitation method. After 10-12 h the medium was changed and cells were incubated for an additional 16-60 h in DMEM-10\% FCS. The medium was again replaced, the retroviral supernatant was harvested and, after removal of cell debris by filtration through $0.25 \mu \mathrm{m}$ cellulose filters, frozen at $-30^{\circ} \mathrm{C}$ for later use.

For retroviral infection, 786-O kidney carcinoma cells $\left(10^{6}\right)$ were plated in $2 \mathrm{ml}$ DMEM supplemented with $10 \%$ FCS in humidified 5\% carbon dioxide atmosphere for $24 \mathrm{~h}$, then incubated with $2 \mathrm{ml}$ BOSC-23 retroviral supernatant supplemented with $8 \mu \mathrm{g} / \mathrm{ml}$ polybrene for $24 \mathrm{~h}$, then again infected with retroviral supernatant supplemented with $8 \mu \mathrm{g} / \mathrm{ml}$ polybrene for additional $8 \mathrm{~h}$. To select for transfected cells, culture medium was then exchanged with $2 \mathrm{ml}$ DMEM-10\% FCS with $5 \mu \mathrm{g} / \mathrm{ml}$ puromycin $(10 \mathrm{mg} / \mathrm{ml})$ per well. Expression of pBABE-construct encoded proteins was confirmed by Western blotting with an antibody against V5, VHL or EGFP, respectively.

Western blot analysis. For preparation of protein extracts, renal tumor tissue was pulverized with a mortar under liquid nitrogen and suspended on ice in lysis buffer (20 mM HEPES, $\mathrm{pH} 7.7,0.2 \mathrm{M} \mathrm{NaCl}, 1.5 \mathrm{mM} \mathrm{MgCl}_{2}, 0.4 \mathrm{mM}$ EDTA, $1 \%$ Triton X-100, $0.5 \mathrm{mM}$ DTT, $100 \mu \mathrm{g} / \mathrm{ml}$ leupeptin, $100 \mu \mathrm{g} / \mathrm{ml}$ aprotinin, $10 \mathrm{mM}$ benzamidine, $2 \mathrm{mM}$ phenylmethylsulphonyl fluoride, $20 \mathrm{mM}$ B-glycerophosphate and $0.1 \mathrm{mM}$ sodiumorthovanadate) (25). Extracts of overall protein were prepared from renal tumor cells by RIPA lysis buffer (10 mM Tris$\mathrm{HCl} \mathrm{pH} 8.0,140 \mathrm{mM} \mathrm{NaCl}, 1 \%$ Triton $\mathrm{X}-100,1 \% \mathrm{Na}-$ deoxycholate, $0.1 \% \mathrm{SDS}, 0.025 \% \mathrm{NaN}_{3}$ ), including protease and phosphatase inhibitors, on ice. Cytoplasmatic proteins were prepared by lysis buffer I (10 mM HEPES/KOH pH 7.9, $1.5 \mathrm{mM} \mathrm{MgCl}_{2}, 10 \mathrm{mM} \mathrm{KCl}, 0.5 \mathrm{mM}$ DTT, $0.1 \%$ bensonase) including protease and phosphatase inhibitors, on ice. After centrifugation, the soluble cytoplasmatic proteins were separated from insoluble nuclei proteins. For preparing nuclei proteins, the pellet was treated by lysis buffer II $(20 \mathrm{mM}$ HEPES/KOH pH 7.9, $1.5 \mathrm{mM} \mathrm{MgCl} 2,420 \mathrm{mM} \mathrm{NaCl}, 0.2 \mathrm{mM}$ EDTA, 25\% glycerol, $0.5 \mathrm{mM}$ DTT, $0.1 \%$ bensonase) including protease and phosphatase inhibitors, on ice. Protein concentrations of the extracts were determined using BCA (bicinchoninic acid) reagents (26).

Equal amounts of the protein extracts (100 $\mu \mathrm{g}$ per lane from cells or $30 \mu \mathrm{g}$ per lane from tissue specimens) were separated by SDS-PAGE (sodium dodecyl sulfate-polyacrylamide) gel electrophoresis of $10 \%$ polyacrylamide and transferred by semi-dry blotting onto polyvinylidene fluoride membranes (PVDF, Immobilon P, Millipore) for Western blotting. The membrane was blocked in Roti-block blocking solution (Roth) for $1 \mathrm{~h}$. The primary antibodies were incubated for one hour in blocking solution. The mouse monoclonal antibodies against V5-TAG (Serotec), VHL (PD Pharmingen), PTEN (25H9), phospho-PTEN Ser380/Thr382/383, phosphoAkt Ser473 (Cell Signaling), phospho-FAK Tyr397 (Millipore), phospho-Shc Tyr 239/240 (Cell Signaling) and B-actin (Sigma-Aldrich) were diluted 1:1000. After washing, the membrane was incubated with horseradish peroxidaseconjugated secondary antibodies (Santa Cruz) and diluted 1:5000 for $1 \mathrm{~h}$ at room temperature. The bound antibodies were visualized by an enhanced chemiluminescence (ECL) detection system using Fuji medical X-ray film.

For quantification of PTEN in tissue specimens, the Western blot membrane was stained with Coomassie brilliant blue (Sigma) and the amount of total protein was calculated by integration of the bands of each lane using Image $\mathbf{J}$ software. To allow comparison of different experiments, the same positive control was loaded on each gel and used as an internal standard. The amount of PTEN was analogously calculated by computer-aided integration of the band after subtraction of the background and referred to the value of total protein.

Statistical analysis. For statistical analyses SPSS 17.0 software was applied. PTEN expression in renal tumor tissue was quantified and presented as relative units. Time of overall and disease specific death of the patients were registered and the 5-year survival rate was calculated. Differences in the expression of PTEN in tumor tissue of patient's dead within 5 years and those surviving were performed using the Mann-Whitney test. Differences were considered statistically significant at $\mathrm{p}<0.05$.

Cell migration assay. A microchemotaxis chamber (Costar) containing an upper and a lower space separated by a porous polycarbonate membrane (pore diameter $8 \mu \mathrm{m}$ ), was employed for the migration experiments. The chamber was divided into 48 wells, resulting in an invasion unit with a surface of $\sim 7.8 \mathrm{~mm}^{2}$. 
Renal tumor cells, transfected as described above, were harvested, washed with PBS and resuspended in serum-free culture medium. In the experiments, the wells of the chemotaxis chamber were coated with $30 \mu \mathrm{l}$ fibronectin, laminin or collagen IV (each $1-500 \mu \mathrm{g} / \mathrm{ml}$ ) and covered with the polycarbonate membrane. The tumor cell suspension $\left(3 \times 10^{5}\right.$ cells/ $\mathrm{ml}$ ) was introduced into the upper part of the chamber. After an incubation period of $16 \mathrm{~h}$ at $37^{\circ} \mathrm{C}$ in a humidified atmosphere containing $5 \% \mathrm{CO}_{2}$ in air (27), cells that did not pass the polycarbonate membrane were removed from the upper side of the porous membrane by washing with buffer solution according to Weise (Merck) and by mechanical removal with a rubber policeman. The membrane was dried for $10 \mathrm{~min}$ and fixed in methanol. Afterwards, the nuclei and cytoplasm were specifically stained with hemacolor (Merck) and embedded on a glass slide coated with immersion oil. The number of invasive tumor cells was evaluated by a microscopic test raster ocular (Zeiss, 400-fold magnification) (28). For a single determination, 10 different views per well with a combined membrane surface of $2.5 \mathrm{~mm}^{2}$ were evaluated. The experiments were performed 4-8-fold and repeated four times. For statistical confirmation, three independent migration experiments, each 4-fold, were performed respectively. A mean value and a standard error were calculated from the results.

To optimize the conditions of the cell migration assay, three compounds of ECM as chemotractants were tested. Fibronectin, laminin and collagen type IV were analyzed in concentrations between 1 and $500 \mu \mathrm{g} / \mathrm{ml}$. Laminin and collagen type IV showed only a marginal chemotactical effect in the migration assay (Fig. 1). The highest migratory effect was observed when using fibronectin as a chemotactical agent in a concentration-dependent manner (Fig. 1). A distinct migration was observed at a concentration $>6 \mu \mathrm{g} / \mathrm{ml}$. Therefore, a concentration of $10 \mu \mathrm{g} / \mathrm{ml}$ was applied in all subsequent experiments.

\section{Results}

Correlation of PTEN expression in ccRCC with survival and development of metastases. To quantify the expression of the tumor suppressor protein PTEN in ccRCC, protein extracts of renal tumor tissue from 144 patients were analysed by Western blotting. PTEN in renal tumor tissue was quantified by computer-aided integration of the bands. The attained values were correlated with survival of the patients. Of the 144 patients analysed, we obtained survival status from 135 patients with a median follow-up period of 50.6 months (minimum, 0.27 month because of the early death of the patient; maximum, 155.2 months). Of these 135 patients, 30 patients died within 5 years after surgery, 22 thereof on account of tumor disease. One hundred and five patients survived the 5-year border. Specific patient data are listed in Table I.

In these 135 patients we quantified PTEN in tumor tissue specimens. We found a correlation between PTEN expression and 5-year disease specific survival as well as overall 5-year survival. In both analyses, we found a $30.1 \%$ reduced PTEN expression (median 0.867 relative units) in patients who died from any cause compared to those patients surviving 5 years after surgery (median 1.254 relative units, Fig. 2). This reduced

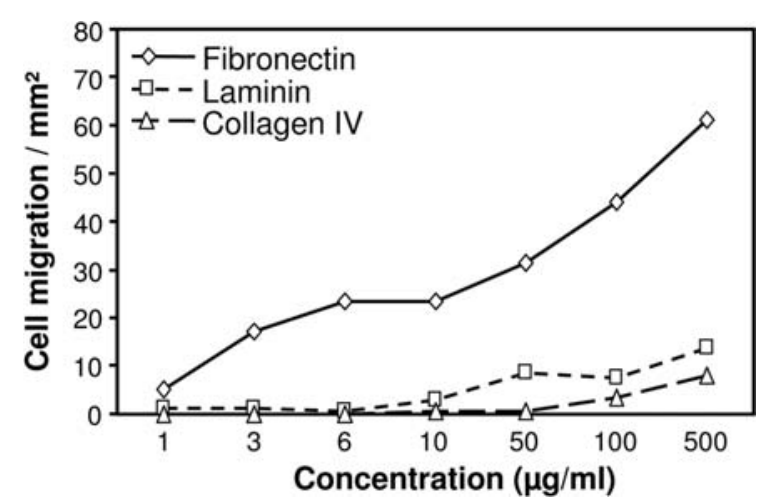

Figure 1. Chemotactical migration of 786-O cells. Fibronectin, laminin or collagen IV in concentrations between 1 and $500 \mu \mathrm{g} / \mathrm{ml}$ were used as chemotaxins. In a Boyden chemotaxis chamber, $\sim 200$ cells $/ \mathrm{cm}^{2}$ were allowed to migrate for $16 \mathrm{~h}$ through a polycarbonate membrane with a pore diameter of $8 \mu \mathrm{m}$. Fibronectin induced the highest migration and was used as chemotaxin for further experiments.

PTEN expression was significant at $\mathrm{p}=0.019$ (Fig. 2A) and 0.002 (Fig. 2B), respectively. These results were independent from histopathological grading or tumor stage.

Influence of PTEN on metastasis in an in vivo mouse model. To verify the relevance of the results obtained in cultured cells, we determined the role of PTEN in a mouse model. We analyzed the development of metastases after injection of wtPTEN transfected cells into the tail vein of athymic mice. As a control for the sensitivity of the cells in the process of metastasis, we used VHL transfected 786-O cells, since we know from our former results and from other groups that VHL expression inhibits cell migration (29). We observed a strongly reduced potential for metastases in both, VHL as well as wtPTEN transfected cells to 3.1 and $2.9 \%$ compared to untransfected 786-O cells, respectively (Fig. 3).

Generation of mutated PTEN isoforms. To examine the role of PTEN in development of metastases, the main reason of death in patients with renal cancer, we analyzed the molecular mechanisms involved in cell migration as the most important step in metastasis. We designed several mutant isoforms to determine the requirement of PTEN's different catalytic activities: point mutation C124E (loss of overall phosphatase activity), point mutation G129E (loss of lipid phosphatase activity) and $\Delta$ Exon 3 (deletion of 14 amino acids in exon 3 , loss of protein phosphatase activity). Additionally, the role of the C-terminal tail was investigated by a deletion of 43 $\mathrm{C}$-terminal amino acids, $\triangle \mathrm{C}$-terminus. In vectors including wtPTEN and PTEN with point mutations, the restriction enzymes SalI and BamHI led to the creation of two fragments of 5127 and $1398 \mathrm{bp}$, as expected. In PTEN constructs with a deletion of the $\mathrm{C}$-terminal tail ( $\Delta$ C-terminus) and exon 3 ( $\Delta$ Exon3), these enzymes resulted in fragments of $5127 / 1197$ or $5127 / 1353 \mathrm{bp}$, respectively, giving evidence for successful deletions. For a further control of the deletion of exon 3, the construct was additionally treated with the restriction enzyme HindIII. This process led to fragments of 4962 and 1518 bp, caused by a newly introduced cut position. Point mutations were verified by sequencing (data not shown). 
Table I. The patient data analyzed by PTEN expression in tumor tissue.

\begin{tabular}{|c|c|c|c|c|}
\hline & \multicolumn{2}{|c|}{ Disease specific survival } & \multicolumn{2}{|c|}{ Overall survival } \\
\hline & $>5$ years & $<5$ years & $>5$ years & $<5$ years \\
\hline Sex (male/female) & $66 / 39$ & $11 / 11$ & $66 / 39$ & $16 / 14$ \\
\hline \multicolumn{5}{|l|}{ Age (years) } \\
\hline median (min-max) & $\begin{array}{c}64.9 \\
(30.6-82.8)\end{array}$ & $\begin{array}{c}63.9 \\
(46.3-86.9)\end{array}$ & $\begin{array}{c}64.9 \\
(30.6-82.8)\end{array}$ & $\begin{array}{c}67.3 \\
(46.3-88.4)\end{array}$ \\
\hline \multicolumn{5}{|l|}{$\mathrm{T}$} \\
\hline 1 & $58(55 \%)$ & $5(23 \%)$ & $58(55 \%)$ & $11(37 \%)$ \\
\hline 2 & $15(14 \%)$ & $4(18 \%)$ & $15(14 \%)$ & $4(13 \%)$ \\
\hline 3 & $31(30 \%)$ & $12(55 \%)$ & $31(30 \%)$ & $14(47 \%)$ \\
\hline 4 & $1 \quad(1 \%)$ & $1 \quad(5 \%)$ & $1 \quad(1 \%)$ & $1(3 \%)$ \\
\hline \multicolumn{5}{|l|}{ G } \\
\hline 1 & $13(12 \%)$ & $4(18 \%)$ & $13(12 \%)$ & $4(13 \%)$ \\
\hline 2 & $61(58 \%)$ & $5(23 \%)$ & $61(58 \%)$ & $11(37 \%)$ \\
\hline $3+4$ & $31(30 \%)$ & $13(59 \%)$ & $31(59 \%)$ & $15(50 \%)$ \\
\hline
\end{tabular}
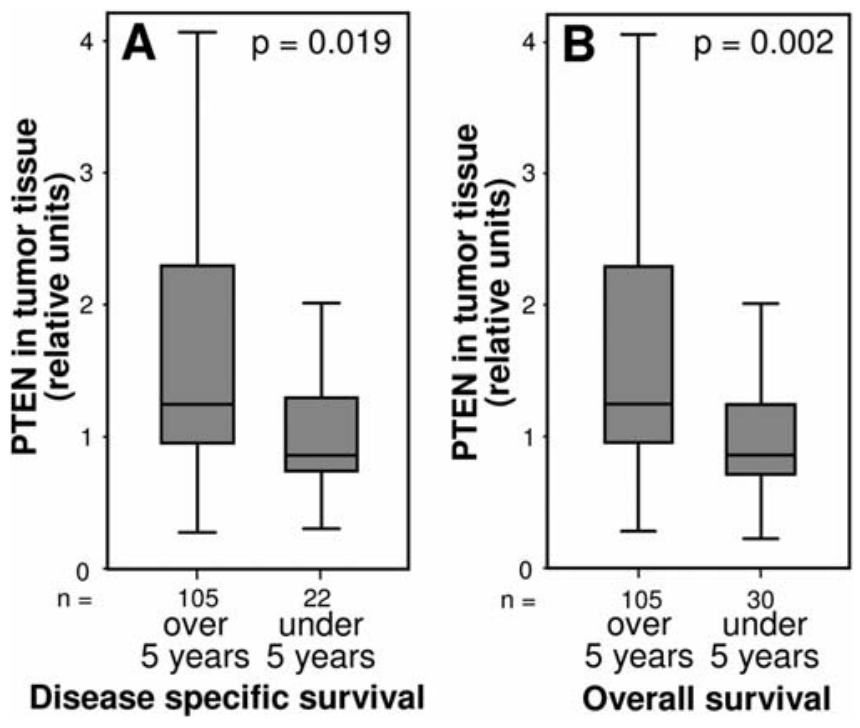

Figure 2. Expression of PTEN in tumors of patients with clear cell renal cell carcinoma. Analyzed were tumor tissue specimens from 135 patients with clear cell RCC by Western blotting. PTEN expression was quantified by densitometry of the specific bands (relative units). Five-years disease specific (A) and overall survival (B) are illustrated for patients surviving and those who died within 5 years after surgery. PTEN expression was significantly higher in the surviving patients than in patients who died within this time period ( $\mathrm{p}=0.019$ and $\mathrm{p}=0.002$, respectively). Box plots show medians (central lane), $25 \%$ and $75 \%$ percentiles (lower and upper side of the box) and minimum and maximum (lower and upper bars). Extreme values are not shown.

Cell transfection. The described PTEN expression constructs and EGFP for control were transduced into the PTEN negative renal cancer cell line $786-\mathrm{O}$ by retroviral transfection. For a specific detection of the gene products of the transfected genes, all constructs were in frame tagged with a V5 encoding sequence, which was localized in 3'-position of the gene

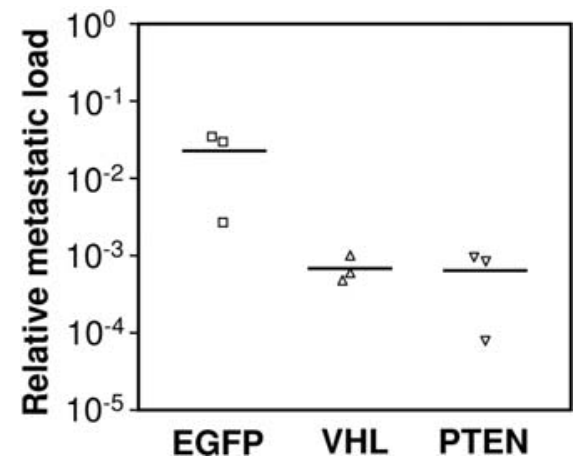

Figure 3. Metastatic load in the lungs of athymic mice after application of $786-\mathrm{O}$ cells. The metastatic load was analyzed 4 weeks after injection of $10^{6}$ 786-O cells transfected with either EGFP, PTEN or VHL into the tail vein. $786-\mathrm{O}$ cells were detected by quantitative real-time PCR of an $\alpha$-satellite region of the human chromosome 17 . Human DNA was determined in ratio to murine DNA (relative metastatic load). Transfection with PTEN resulted in a strongly reduced metastatic load in the same order of magnitude as transfection with the functional positive control, VHL.

(PTEN-V5). After transfection, protein extracts of the cells were analyzed by Western blotting with a V5 specific antibody. In the renal cancer cell line 786-O, all transfectants showed expression of V5 in the cytosolic as well as the nuclear fraction in the expected molecular weight (Fig. 4). These results show a successful transfection of all constructs of PTEN in the renal cancer cells.

Migration of PTEN transfected renal cancer cells. VHL transfected 786-O cells, used as a control for the sensitivity of the cells for migration inhibitory effects (29), showed a reduced migration down to $80.0 \%$ of control cells transfected with EGFP (Fig. 5). After transfection of the cells with 


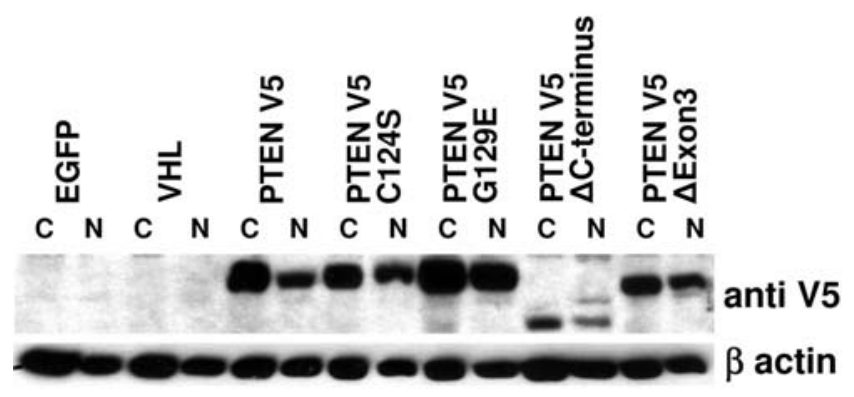

Figure 4. Expression of PTEN in renal cancer cells transfected with several genes. The 786-O cells transfected with EGFP (control), VHL or mutated PTEN genes by Western blotting from cytosolic (C) or nuclear $(\mathrm{N})$ protein extracts were analyzed. PTEN mutants were tagged with V5. Detection of transfected PTEN isoforms was performed with an anti-V5 antibody. Loading control was done with an anti- $\beta$ actin antibody. In all cells transfected with a PTEN isoform, PTEN was detectable. The band of PTEN $\triangle \mathrm{C}$-terminus and PTEN $\triangle$ exon 3 showed lower molecular weight because of the encoded deletions leading to 43 and 14 amino acid-reduced molecules, respectively. In cells transfected with EGFP (control) or VHL no PTEN was detectable.

wtPTEN, cell migration was reduced down to $45.0 \%$ of control. This effect was also observed by transfecting the cells with PTEN G129E (45.3\% of control), leading to a reduced lipid phosphatase activity of PTEN, and PTEN $\triangle \mathrm{C}$-terminus (loss of the C-terminal tail, $47.3 \%$ of control). In contrast, after transfection with PTEN C124S (reduced overall phosphatase activities of PTEN), this effect was partly abolished (87.0\% of control). A deletion of exon 3 in the PTEN gene (PTEN $\triangle$ Exon3, loss of protein phosphatase activity) did not result in reduced migration (133.3\%) compared to the PTEN deficient control (Fig. 5). These results show that PTEN reduces the chemotactical cell migration in 786-O cells, and that in particular its protein phosphatase activity is responsible for this effect.

Analysis of PTEN downstream activities. The results described show a critical role of the protein phosphatase activity of PTEN in cell migration. To verify the functional activity of PTEN molecules transfected in 786-O cells, we analyzed the activity conditions of the PTEN downstream targets Akt, FAK and Shc by expression of their phosphorylated versions. Dependent on the PTEN isoform transfected, we observed different expression patterns of Akt phosphorylated in position Ser473 (Fig. 6A). In control cells and cells transfected with VHL, phospho-Akt was observed. After transfection with wtPTEN or PTEN $\triangle C$-teminus, phospho-Akt disappeared. In contrast, transfection with PTEN C124S or PTEN G129E led to no reduction of phospho-Akt. A deletion of exon 3 (PTEN Dexon3) resulted in a marginally reduced expression of phospho-Akt (Fig. 6A). The reduced amount of phosphoAkt after transfection with PTEN isoforms without an overallor lipid phosphatase activity confirmed the successful inactivation of the PTEN lipid phosphatase activity by the used mutated isoforms.

The expression of phosphorylated FAK (Tyr397) was unchanged in all wild-type and mutated PTEN (Fig. 6B). In contrast, the expression of Shc phosphorylated in positions Tyr239/240 differed depending on the transfected PTEN molecule. After transfection with wtPTEN, PTEN G129E (loss of lipid phosphatase activity) and PTEN $\triangle C$-terminus, the amount of phospho-Shc was clearly reduced. In contrast, transfection of PTEN genes without protein phosphatase activity (PTEN C124S and PTEN $\triangle E x o n 3$ ) quantity of phospho-Shc was similar to the amount in EGFP control cells.

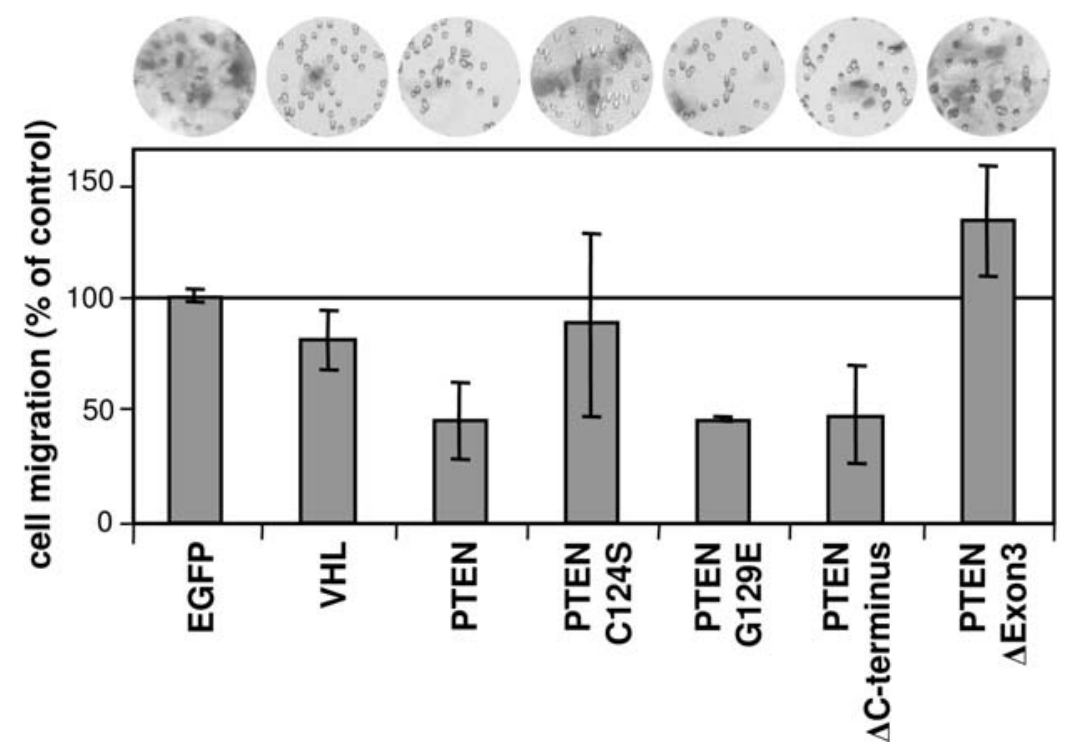

Figure 5. Cell migration of transfected 786-O cells. Transfections were performed with EGFP (control), VHL (functional positive control) or mutated PTEN isoforms. Cell migration was determined in a Boyden chemotaxis chamber. Approximately $200 \mathrm{cells}^{\mathrm{s}} / \mathrm{cm}^{2}$ were allowed to migrate for $16 \mathrm{~h}$ through a polycarbonate membrane with a pore diameter of $8 \mu \mathrm{m}$. The mean values and standard errors (SEM) in percent of control of 3-4 independent experiments, each 4-fold are demonstrated. Above each bar a microscopic section of the porous polycarbonate membrane including migrated cells (blue staining) is demonstrated. Mutations of PTEN lead to the following effects: PTEN C124S, loss of overall phosphatase activity; PTEN G129E, loss of lipid phosphatase activity; PTEN $\triangle$ exon3, loss of protein phosphatase activity; PTEN $\triangle \mathrm{C}$-terminus, loss of PDZ binding domain and phosphorylation sites. Functional PTEN reduced cell migration, which was abolished after interrupting the protein phosphatase activity. 

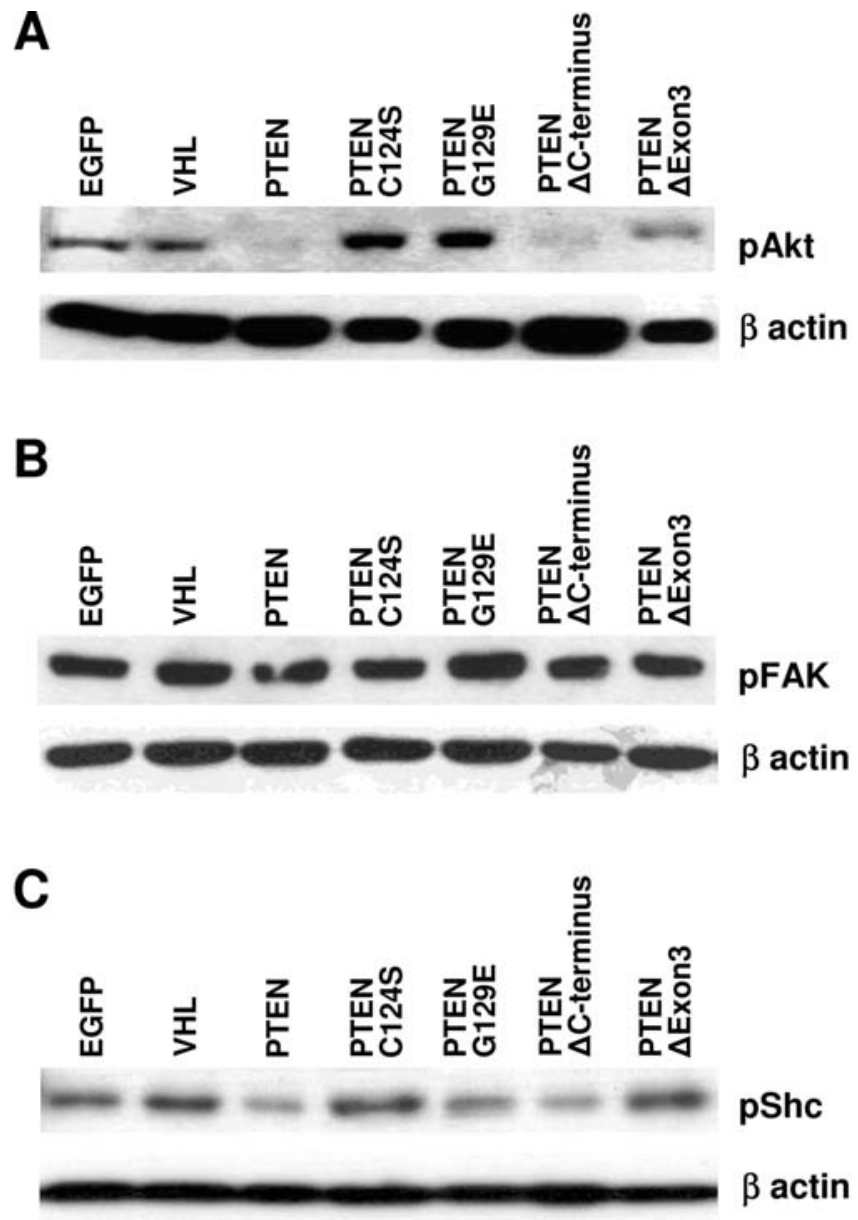

Figure 6. Phosphorylation status of PTEN downstream targets. (A) Akt, (B) FAK and (C) Shc in 786-O cells after transfection with EGFP (control), $V H L$ or mutated PTEN isoforms were analyzed. Expression of phosphorylated proteins was determined by Western blotting $(100 \mu \mathrm{g}$ protein per lane) specific for the phosphorylated antigens. ß-actin was used as loading control. In transfectants with intact lipid phosphatase activity of PTEN (PTEN, PTEN $\triangle \mathrm{C}$-terminus and PTEN $\triangle$ exon3), expression of phosphorylated Akt was reduced. Expression of phosphorylated FAK was unchanged in all transfectants. Expression of phosphorylated Shc was reduced in cases of PTEN with protein phosphatase activity (PTEN, PTEN G129E and PTEN $\triangle$ C-terminus) and unchanged in case of PTEN with loss of protein phosphatase activity (PTEN C124S and PTEN $\Delta$ exon3).

\section{Discussion}

The tumor suppressor PTEN is known to be involved in the regulation of proliferation, apoptosis and cell motility. In the present study we addressed the role of PTEN in processes of metastases in clear cell renal cancer. In our analyses of native clear cell RCC tissue of 135 patients we found a significant correlation between PTEN expression in tumor tissue and the 5-year disease specific survival, as well as overall survival of the patients. Patients who died within 5 years after surgery showed a $30 \%$ reduced PTEN expression compared to those who survived 5 years. Since death in renal cancer is usually caused by distant metastases, disease specific death at least in these cases should be attributed to the development of metastases. However, for most patients, we have no information on the date of metastases development, but the date of death, so that our analyses concentrated on patient survival. Our analyses suggest that patients with an increased level of
PTEN in tumor tissue have a lower risk for developing metastases. Merseburger and coworkers observed a correlation between PTEN expression and status of metastasis in renal cancer. In contrast to our results, the same authors detected no correlation between PTEN expression and survival. However, they determined the presence of metastases at the time of tumor diagnosis but not during follow-up (30). Other groups showed a reduced disease specific survival in RCC patients expressing low levels of PTEN $(31,32)$, corresponding to our results. However, all these studies were performed by immunohistochemical staining of general RCC without differenciation between histological subtypes. Our analysis was restricted to clear cell RCC, the most frequent and malignant RCC (33) and the presented Western blot analyses quantified the analysed proteins more accurately. The correlation between higher PTEN quantity and diminished occurrence of metastases agrees with the finding that an enhanced in vitro expression of PTEN leads to reduced cell migration and invasion $(21,34,35)$.

To test the significance of our in vitro findings, we injected 786-O cells transfected with the functional PTEN or VHL genes into the tail vein of athymic mice and calculated the metastatic load of the lungs in comparison to injected unmodified PTEN negative 786-O cells. We found a strongly reduced metastatic load after PTEN expression, in the same order of magnitude as after transfection with $V H L$. This finding confirms our clinical results that PTEN expression in ccRCC cells correlates with development of metastases and shows the profundity of our results determined in vitro.

Based on these observations we aimed to find out which enzymatic activity of PTEN is responsible for migration and subsequent metastases. Using mutated PTEN isoforms transfected into PTEN negative 786-O cells, we differentiated between general phosphatase activity, lipid phosphatase activity, protein phosphatase activity and the role of the Cterminal tail of PTEN. Analyses in a Boyden chemotaxis chamber demonstrated that migration of the renal carcinoma cells is obviously regulated by the protein phosphatase activity of PTEN. We observed an inhibitory effect of functional PTEN on cell migration, which was abolished after abrogating the protein phosphatase activity. Inactivation of the lipid phosphatase activity of PTEN alone or deletion of the Cterminal tail did not influence the inhibitory function of PTEN on cell migration (Fig. 5).

The role of the PDZ binding domain in the C-terminus of PTEN is controversially discussed. Whereas some studies demonstrated the importance of the PDZ binding domain in PTEN activity (7), other authors showed a PDZ independent PTEN activity. Yet it seems reasonable that loss of the PDZ binding domain should constrain the translocation of PTEN and so inhibit its activity. Leslie et al demonstrated that expression of PTEN lacking the PDZ binding domain resulted in a loss of cell polarity and migration of mesoderm cells (18). In contrast, Das and coworkers demonstrated an unchanged localization of PTEN to the membrane after deletion of the C-terminus of PTEN (36) and Mosessian and coworkers also showed that the PDZ binding domain is not required for the complex binding of PTEN with its substrates (37). The loss of the phosphorylation sites at the C-terminus 
has an additional effect on the activity of PTEN. Phosphorylation of PTEN results in an interaction of the C-terminus with the phosphatase domain and leads to an inactivation of PTEN (38). Additionally, in this conformation of PTEN, the PDZ binding domain is masked (8). However, reduced localization of PTEN to the membrane is not caused by PDZ masking, but by blocking of the PTEN electrostatic membrane binding (36). Therefore deletion of the C-terminus with the resulting loss of the phosphorylation sites, should lead to a constitutive active PTEN molecule, since the blocking effect by masking the phosphatase domain is abolished (3). However, in our study deletion of the C-terminus resulted in an unchanged lipid and protein phosphatase activity of PTEN in 786-O cells, according to the activity of downstream targets of PTEN. Cell migration was reduced in the same manner after transfection with wtPTEN. We therefore conclude that the role of the C-terminus of PTEN, including the PDZ domain and phosphorylation sites, is probably dependent on the cell type.

FAK, the adapter molecule Shc (39) and Akt are downstream targets of the phosphatase activity of PTEN and are known to be crucial for regulation of cell migration. After binding of integrins to compounds of the extracellular matrix autophosphorylation induces consequently the activation of FAK by phosphorylation on Tyr397. This modification is responsible for promoting signal transduction downstream of FAK and influences cell motility (40). Shc as an adaptor molecule activating Raf and the MAPK signaling pathway also influences cell migration via a phosphorylation event. Phosphorylated at position Tyr239/240 it induces Rho-GTPasedependent cytoskeletal reorganisations, leading to enhanced cell motility $(19,41)$. Akt, activated after phosphorylation at position Ser473 (42), is the most important downstream target of PTEN and influences cell migration by several mechanisms. For example, APE (Akt phosphorylation enhancer) is phosphorylated by Akt, inducing the formation of lamellipodia and leading to cell migration (43). A further substrate of Akt involved in cell migration is ACAP1 (ADPribosylation factor directed GTPase-activating protein 1), which is involved in integrin recycling and increases cell migration (44). Furthermore, Rac and Cdc42 are activated by Akt and relieve cell migration by cytoskeletal rearrangement (45).

Our analyses of the PTEN substrates FAK Tyr397 and Shc Tyr239/240 revealed that in ccRCC cells the amount of phospho-FAK was unchanged in all transfectants with all PTEN isoforms, suggesting that functional PTEN does not directly lead to a reduction of phospho-FAK, as predicted by others $(21,39)$. Liliental and coworkers observed the same unchanged FAK phosphorylation behaviour in fibroblasts transfected with the wtPTEN gene (34). In contrast to these findings, ccRCC cells transfected with a protein phosphatase active PTEN isoform, phospho-Shc was reduced. Complementary, in assays with protein phosphatase deficient PTEN isoforms, phospho-Shc expression was unchanged, compared to the PTEN deficient control. These results indicate that the reduced inhibitory effect on migration, found after transfection with protein phosphatase deficient PTEN genes, is caused by inhibition of Shc dephosphorylation. Quantification of phospho-Akt (Akt Ser473) as a monitor for Akt signalling activity, as expected, showed in the ccRCC cells transfected with lipid phosphatase deficient PTEN isoforms detectable amounts of phospho-Akt. In cells harbouring a PTEN isoform with functional lipid phosphatase domain, phospho-Akt was clearly reduced or even absent.

In conclusion, the protein phosphatase activity of PTEN in ccRCC cells is mainly responsible for reduction of phosphoShc and the lipid phosphatase domain seems to diminish phospho-Akt. The most efficient blocking of cell migration is observed in ccRCC cells transfected with wtPTEN or an isoform with deficient lipid phosphatase domain and with deletion of the C-terminal tail, but not with isoforms harbouring a reduced overall phosphatase activity of PTEN or loss of protein phosphatase activity. We therefore conclude that the protein phosphatase ability of PTEN seems to control cell migration via the enzymatic cascade involving Shc.

\section{Acknowledgements}

This work was supported by the Priority Program Minimal Invasive Surgery.

\section{References}

1. Leslie NR and Downes CP: PTEN function: how normal cells control it and tumour cells lose it. Biochem J 382: 1-11, 2004.

2. Sulis ML and Parson R: PTEN: from pathology to biology. Trenda Cell Biol 13: 478-483, 2003.

3. Vazquez F, Ramaswamy S, Nakamura N and Sellers WR: Phosphorylation of the PTEN tail regulates protein stability and function. Mol Cell Biol 20: 5010-5018, 2000.

4. Georgescu MM, Dirsch KH, Kaloudis P, Yang H, Pavietich NP and Hannafusa $\mathrm{H}$ : Stabilization and productive positioning roles of the C2 domain of PTEN tumor suppressor. Cancer Res 60: 7033-7038, 2000.

5. Wu X, Hepner K, Castelino-Prabhu S, Do D, Kaye MB, Yuan XJ, Wood J, Ross C, Sawyers CL and Whang YE: Evidence for regulation of the PTEN tumor suppressor by a membranelocalized multi-PDZ domain containing scaffold protein MAGI-2. Proc Natl Acad Sci USA 97: 4233-4238, 2000.

6. Adey NB, Huang L, Ormonde PA, Baumgard ML, Pero R, Byreddy DV, Tavtigian SV and Bartel PL: Threonine phosphorylation of the MMAC1/PTEN PDZ binding domain both inhibits and stimulates PDZ binding. Cancer Res 60: 35-37, 2000.

7. Valiente M, Andrés-Pons A, Gomar B, Torres J, Gil A, Tapparel C, Antonarakis SE and Pulido R: Binding of PTEN to specific PDZ domains contributes to PTEN protein stability and phosphorylation by microtubule-associated serine/threonine kinases. J Biol Chem 280: 28936-28943, 2005.

8. Vazquez F, Grossman SR, Takahashi Y, Rokas MV, Nakamura N and Sellers WR: Phosphorylation of the PTEN tail acts as an inhibitory switch by preventing its recruitment into a protein complex. J Biol Chem 276: 48627-48630, 2001.

9. Li Li J, Yen C, Liaw D, Podsypanina K, Bose S, Wang SI, Puc J, Miliaresis C, Rodgers L, McCombie R, Bigner SH, Giovanella BC, Ittmann M, Tycko B, Hibshoosh H, Wigler MH and Parsons R: PTEN, a putative protein tyrosine phosphatase gene mutated in human brain, breast, and prostate cancer. Science 275: 1943-1947, 1997.

10. Guldberg P, Thor-Straten P, Birck A, Ahrenkiel V, Kirkin AF and Zeuthen J: Disruption of the MMAC1/PTEN gene by deletion or mutation is a frequent event in malignant melanoma. Cancer Res 57: 3660-3663, 1997.

11. Bonneau D and Longy M: Mutations of the human PTEN gene. Hum Mutat 16: 109-122, 2000.

12. Beaudet AL and Tsui C: A suggested nomenclature for designating mutations. Hum Mutat 2: 245-248, 1993.

13. Kurose K, Bando K, Fukino K, Sugisaki Y, Araki T and Emi M: Somatic mutations of the PTEN/MMAC1 gene in fifteen Japanese endometrial cancers: evidence for inactivation of both alleles. Jpn J Cancer Res 89: 842-848, 1998.

14. Risinger JI, Hayes AK, Berchuck A and Barrett JC: PTEN/MMAC1 mutations in endometrial cancers. Cancer Res 57: 4736-4738, 1997. 
15. Agrawal S, Pilarski R and Eng C: Different splicing defects lead to differential effects downstream of the lipid and protein phosphatase activities of PTEN. Hum Mol Genet 14: 2459-2468, 2005.

16. Maehama T and Dixon JE: The tumor suppressor, PTEN/ MMAC1, dephosphorylates the lipid second messenger, phosphatidylinositol 3,4,5-trisphosphate. J Biol Chem 273: 13375-13378, 1998.

17. Cully M, You H, Levine AJ and Mak TW: Beyond PTEN mutations: the PI3K pathway as an integrator of multiple inputs during tumorigenesis. Nat Rev Cancer 6: 184-192, 2006.

18. Leslie NR, Yang X, Downes CP and Weijer CJ: PtdIns(3,4,5)P(3)dependent and -independent roles for PTEN in the control of cell migration. Curr Biol 17: 115-125, 2007.

19. Gu J, Tamura M, Pankov R, Danen EH, Takino T, Matsumoto K and Yamada KM: Shc and FAK differentially regulate cell motility and directionality modulated by PTEN. J Cell Biol 146: 389-403, 1999.

20. Mahimainathan L and Choudhury GG: Inactivation of plateletderived growth factor receptor by the tumor suppressor PTEN provides a novel mechanism of action of the phosphatase. J Biol Chem 279: 15258-15268, 2004.

21. Tamura M, Gu J, Matsumoto K, Aota S, Parsons R and Yamada KM: Inhibition of cell migration, spreading, and focal adhesions by tumor suppressor PTEN. Science 280: 1614-1617, 1998.

22. Raftopoulou M, Etienne-Manneville S, Self A, Nicholls S and Hall A: Regulation of cell migration by the $\mathrm{C} 2$ domain of the tumor suppressor PTEN. Science 303: 1179-1181, 2004.

23. Iliopoulos O, Kibel A, Gray S and Kaelin WG Jr: Tumour suppression by the human von Hippel-Lindau gene product. Nat Med 1: 822-826, 1995.

24. Brenner W, Färber G, Herget T, Lehr H-A, Hengstler JG and Thüroff JW: Loss of the tumor suppressor protein PTEN during renal carcinogenesis. Int J Cancer 99: 53-57, 2002.

25. Pear WS, Nolan GP, Scott ML and Baltimore D: Production of high-titer helper-free retroviruses by transient transfection. Proc Natl Acad Sci USA 90: 8392-8396, 1993.

26. Smith PK, Krohn RI, Hermanson GT, Malia AK, Gartner FH, Provenzano MD, Fujimato EK, Golke NM, Olsen BJ and Klenk DC: Measurement of protein using bicinchoninic acid Anal Biochem 150: 76-85, 1985.

27. Brenner W, Gross S, Steinbach F, Horn S, Hohenfellner R and Thüroff JW: Differential inhibition of renal cancer cell invasion mediated by fibronectin, collagen IV and laminin. Cancer Lett 155: 199-205, 2000.

28. Brenner W, Hempel G, Steinbach F, Hohenfellner R and Thüroff JW: Enhanced expression of ELAM-1 on endothelium of renal cell carcinoma compared to the corresponding normal renal tissue. Cancer Lett 143: 14-21, 1999.

29. Zia MK, Rmali KA, Watkins G, Mansel RE and Jiang WG: The expression of the von Hippel-Lindau gene product and its impact on invasiveness of human breast cancer cells. Int J Mol Med 20: 605-611, 2007.

30. Merseburger AS, Hennenlotter J, Kuehs U, Simon P, Kruck S, Koch E, Stenzl A and Kuczyk MA: Activation of PI3K is associated with reduced survival in renal cell carcinoma. Urol Int 80: 372-377, 2008
31. Pantuck AJ, Seligson DB, Klatte T, Yu H, Leppert JT, Moore L, O'Toole T, Gibbons J, Belldegrun AS and Figlin RA: Prognostic relevance of the mTOR pathway in renal cell carcinoma: implications for molecular patient selection for targeted therapy. Cancer 109: 2257-2267, 2007.

32. Kim HL, Seligson D, Liu X, Janzen N, Bui MH, Yu H, Shi T, Belldegrun AS, Horvath S and Figlin RA: Using tumor markers to predict the survival of patients with metastatic renal cell carcinoma. J Urol 173: 1496-1501, 2005.

33. Buentig N, Storkel S and Atzpodien J: Molecular genetic changes in renal cell carcinomas. Urologe A 41: 475-481, 2002.

34. Liliental J, Moon SY, Lesche R, Mamillapalli R, Li D, Zheng Y, Sun $\mathrm{H}$ and $\mathrm{Wu} \mathrm{H}$ : Genetic deletion of the Pten tumor suppressor gene promotes cell motility by activation of Rac1 and $\mathrm{Cdc} 42$ GTPases. Curr Biol 10: 401-404, 2000.

35. Sanchez T, Thangada S, Wu MT, Kontos CD, Wu D, Wu H and Hla T: PTEN as an effector in the signaling of antimigratory $\mathrm{G}$ protein-coupled receptor. Proc Natl Acad Sci USA 102: 4312-4317, 2005.

36. Das S, Dixon JE and Cho W: Membrane-binding and activation mechanism of PTEN. Proc Natl Acad Sci USA 100: 7491-7496, 2003.

37. Mosessian S, Avliyakulov NK, Mulholland DJ, Boontheung P, Loo JA and Wu H: Analysis of PTEN complex assembly and identification of heterogeneous nuclear ribonucleoprotein $\mathrm{C}$ as a component of the PTEN-associated complex. J Biol Chem 284: 30159-30166, 2009.

38. Odriozola L, Singh G, Hoang T and Chan AM: Regulation of PTEN activity by its carboxyl-terminal autoinhibitory domain. J Biol Chem 282: 23306-23315, 2007.

39. Gu J, Tamura M and Yamada KM: Tumor suppressor PTEN inhibits integrin- and growth factor-mediated mitogen-activated protein (MAP) kinase signaling pathways. J Cell Biol 143: 1375-1383, 1998

40. Choma DP, Milano V, Pumiglia KM and Di Persio CM: Integrin alpha3beta1-dependent activation of FAK/Src regulates Rac1-mediated keratinocyte polarization on laminin-5. J Invest Dermatol 127: 31-40, 2007.

41. Yamazaki D, Kurisu S and Takenawa T: Regulation of cancer cell motility through actin reorganization. Cancer Sci 96: 379-386, 2005.

42. Sarbassov DD, Guertin DA, Ali SM and Sabatini DM: Phosphorylation and regulation of Akt/PKB by the rictor-mTOR complex. Science 307: 1098-1101, 2005.

43. Enomoto A, Murakami H, Asai N, Morone N, Watanabe T, Kawai K, Murakumo Y, Usukura J, Kaibuchi K and Takahashi M: Akt/PKB regulates actin organization and cell motility via Girdin/APE. Dev Cell 9: 389-402, 2005.

44. Li J, Ballif BA, Powelka AM, Dai J, Gygi SP and Hsu VW: Phosphorylation of ACAP1 by Akt regulates the stimulationdependent recycling of integrin beta1 to control cell migration. Dev Cell 9: 663-673, 2005.

45. Yamada KM and Araki M: Tumor suppressor PTEN: modulator of cell signaling, growth, migration and apoptosis. J Cell Sci 114: 2375-2382, 2001 